\title{
Glycemic index and glycemic load are associated with some cardiovascular risk factors among the PREMIER study participants
}

\author{
Pao-Hwa Lin'*, Chuhe Chen², Deborah R. Young ${ }^{3}$, \\ Diane Mitchell, ${ }^{4}$ Patricia Elmer ${ }^{2}$, Yanfang Wang ${ }^{5}$, Bryan Batch ${ }^{6}$ \\ and Catherine Champagne ${ }^{7}$
}

\begin{abstract}
'Department of Medicine, Nephrology Division, Duke University Medical Center, Durham, NC, USA; ${ }^{2}$ Kaiser Permanente Center for Health Research, Portland, OR, USA; ${ }^{3}$ Department of Epidemiology and Biostatistics, University of Maryland School of Public Health, College Park, MD, USA; ${ }^{4}$ The Diet Assessment Center, Pennsylvania State University, University Park, PA, USA; ${ }^{5}$ Clinical Research Institute, Peking University Health Science Center, Beijing, China; ${ }^{6}$ Department of Medicine, Endocrinology Division, Duke University Medical Center, Durham, NC, USA;

${ }^{7}$ Pennington Biomedical Research Center, Louisiana State University System, Baton Rouge, LA, USA
\end{abstract}

\section{Abstract}

Background: The clinical significance of glycemic index (GI) and glycemic load (GL) is inconclusive.

Objective: This study was conducted to examine the association of GI and GL with clinical cardiovascular disease (CVD) risk factors including body weight, blood pressure (BP), serum lipids, fasting glucose, insulin and homocysteine over time among the PREMIER participants.

Design: PREMIER was an 18-month randomized lifestyle intervention trial, conducted from 2000 to 2002, designed to help participants reduce BP by following the Dietary Approaches to Stop Hypertension (DASH) dietary pattern, losing weight, reducing sodium and increasing physical activity. GI and GL were estimated from 24 h diet recall data at baseline, 6 and 18 months after intervention. PROC MIXED model was used to examine the association of changes in GI or GL with changes in CVD risk factors.

Results: A total of 756 randomized participants, 62\% females and 34\% African Americans and who averaged $50.0 \pm 0.3$ years old and $95.3 \pm 0.7 \mathrm{~kg}$, were included in this report. Neither GI nor GL changes was associated with changes in any risk factors at 6 months. At 18 months, however, the GI change was significantly and positively associated with total cholesterol (TC) change only $(p<0.05, \beta=23.80 \pm 12.11 \mathrm{mg} / \mathrm{dL}$ or $0.62 \pm 0.31$ $\mathrm{mmol} / \mathrm{L})$ with a significant age interaction. The GL change was significantly associated with $\mathrm{TC}(p=0.02$, $\beta=0.28 \pm 0.15 \mathrm{mg} / \mathrm{dL}$ or $0.01 \pm 0.00 \mathrm{mmol} / \mathrm{L}$ ) positively and with low density lipoprotein cholesterol (LDL-C) changes negatively $(p=0.03, \beta=-0.01 \pm 0.00 \mathrm{mg} / \mathrm{dL}$ or $-0.00 \pm 0.00 \mathrm{mmol} / \mathrm{L}$ ), and significant age interactions were observed for both.

Conclusion: GI and GL was associated with TC and LDL-C after controlling for energy, fat and fiber intake and other potential confounders and the associations were modified by age. Further investigation into this relationship is important because of its potential clinical impact.

Keywords: glycemic index; glycemic load; diet; cardiovascular risk

Received: I4 September 20II; Revised: 24 February 2012; Accepted: 17 April 2012; Published: 4 June 2012

$\mathrm{S}$ ince 1981 the glycemic index (GI) has been used to rank foods based on their effect on postprandial glycemia (1). The concept of glycemic load (GL), which incorporates the amount of carbohydrates consumed into the equation, was later developed to further quantify the glycemic effect of foods. Numerous studies have examined the potential physiological impact of GI and GL but their clinical significance remains debatable. Some cross-sectional or prospective observational studies show an association between GI and GL with cardiovascular disease (CVD) risk factors (2-4), but not all $(5,6)$. Randomized controlled trials have also shown 
inconsistent associations (7-9). In addition, several studies have shown that a reduced GI or GL is associated with a reduced risk for CVD, coronary heart disease and diabetes mellitus $(2,3,10,11)$. However, other studies have not reported such an association $(5,12,13)$. Overall, results from studies examining the association between GI and/or GL and CVD risk factors including blood pressure (BP), insulin and lipids have not been consistent. Further, to date, relatively little information has been published on the long-term effects of changes in dietary GI or GL with changes in CVD risk markers. The PREMIER study offered an opportunity to examine these associations more fully because of its longer term design and the substantial number of participants recruited. The primary objective of this paper is to examine the association of GI and GL with clinical CVD risk factors including body weight, BP, serum lipids, fasting glucose, insulin and homocysteine over 18 months period of time among the PREMIER participants. Even though the causal relationship between homocysteine and CVD has not been established yet, many studies have shown an association between this marker and CVD risk (14). In addition, research has also suggested that glycemic control, such as in the expression of insulin secretion, may affect homocysteine level (15). Thus, this marker is also included in the current analysis.

\section{Methods}

\section{Study design}

PREMIER was a randomized clinical trial, conducted from 2000 to 2002, designed to determine the effects of two multi-component lifestyle interventions on BP. Detailed descriptions of the study design, the intervention programs and the main results have been published elsewhere $(16,17)$. Participating institutions included the National Heart Lung and Blood Institute (NHLBI) Project Office [Bethesda, MD], the Coordinating Center [Kaiser Permanente Center for Health Research in Portland, OR] and four clinical centers [Duke University Medical Center, Durham, NC; Johns Hopkins University, Baltimore, MD; Pennington Biomedical Research Center, Baton Rouge, LA; and Kaiser Permanente Center for Health Research, Portland, OR]. Institutional review boards at each center and an external protocol review committee approved the protocol. Each participant provided written informed consent.

\section{Study participants}

A total of 810 participants were recruited and randomized into the study. Individuals were eligible if they were age 25 or older, had a BMI of $18-45 \mathrm{~kg} / \mathrm{m}^{2}$, were not taking anti-hypertensive medication and had a systolic BP (SBP) of 120-159 mmHg and diastolic BP (DBP) of $80-95 \mathrm{mmHg}$, based on the mean BP over three screening visits. Major exclusion criteria were regular use of drugs that affect BP, including the Sixth Report of the Joint National Committee on Prevention, Detection, Evaluation, and Treatment of High Blood Pressure (JNC-VI) risk category $\mathrm{C}$ [target organ damage and/or diabetes], use of weight-loss medications, prior cardiovascular event, heart failure, angina, cancer diagnosis or treatment in the past 2 years, consumption of $>21$ alcoholic drinks/ week, and pregnancy, planned pregnancy, or lactation.

\section{Intervention}

After eligibility was established, study participants were randomly assigned to one of three intervention groups: (1) a behavioral lifestyle intervention that implemented established recommendations [EST], (2) a behavioral lifestyle intervention that implemented established recommendations plus the DASH dietary pattern [ESTDASH], or (3) an advice only control group. The intervention lasted a total of 18 months, with 6 months of intensive intervention and 12 months of maintenance. Both EST and EST-DASH participants received weekly group sessions for the first 8 weeks, then bi-weekly through the remainder of the first 6 months and monthly for the last 12 months. Seven individual sessions were interspersed throughout the 18 months. Trained and certified interventionists, generally registered dietitians, conducted all the intervention sessions.

Participants' lifestyle goals for both the EST and ESTDASH interventions were weight loss of at least $6.8 \mathrm{~kg}$ (15 lb) at 6 months for those with a BMI $\geq 25 \mathrm{~kg} / \mathrm{m}^{2}$, at least $180 \mathrm{~min} /$ week of moderate-intensity physical activity, no more than $100 \mathrm{mmol} /$ day of dietary sodium, and no more than $30 \mathrm{ml}(1 \mathrm{oz}) /$ day of alcohol [two drinks] for men and $15 \mathrm{ml}(1 / 2 \mathrm{oz}) /$ day [one drink] for women. In addition, individuals in the EST-DASH intervention were counseled to implement the DASH dietary pattern, with the following goals: 9-12 servings of fruits and vegetables, 2-3 servings of low-fat dairy products per day, and intake of total and saturated fat of no more than 25 and $7 \%$ of total calories, respectively.

In contrast, the advice only control group received a single 30 min individual advice session at the time of randomization. At that session, participants received verbal instruction, and written materials that provided information on established recommendations and the DASH dietary pattern. However, no behavioral counseling or further intervention contact was provided until after completion of the 6-month data collection.

\section{Measurements}

All measurements were obtained at baseline, 6 and 18 months after randomization by staff who were blinded to randomization assignment. Intake of nutrients and food groups were assessed from unannounced 24-h dietary recalls conducted by telephone interviews. 
Two recalls were collected by the Diet Assessment Center of the Pennsylvania State University at each study time point [one week day and one weekend day] using the Nutrition Data System for Research (NDS-R) (Nutrition Coordinating Center, University of Minnesota, MN). Across the four clinical sites, the completion rate of the diet recall averaged over $90 \%$. Average of the two recalls for each time point was used for the analysis in this report. For this report, dietary intake files were recalculated using Nutrition Data System for Research (NDS-R) (Nutrition Coordinating Center, University of Minnesota, Minneapolis, MN, version 2006) which contains values for GI and GL in addition to updates to the nutrient and food group files since the 1998 release of NDS-R. GI values for daily totals, recipes and formulations in the NDSR are calculated from the GI and weighted by available carbohydrate of each ingredient food. For foods where measured GI data were unavailable in the literature, GI was either estimated from similar foods, calculated from available carbohydrate amounts and the GI of ingredients within the food, or given a default GI. Methodology for selection of GI values and their incorporation into the database has been reported elsewhere (18). NDS-R has a time-related feature that enables the recalculation of data from previous versions of the original data collected. GI and GL values were adjusted for energy intake using the residual method (19).

Fasting blood was collected for the measurement of a lipid profile, insulin, glucose and homocysteine (only available for the baseline and 6-month visits) at a central lab (Washington University, St. Louis) (20) following standardized protocols. Lipid profile was assessed by Hitachi 917 and glucose by Roche hexokinase method on Hitachi 917, and insulin by Roche Elecsys 2010 (Roche Diagnostics, Indianapolis, IN). The coefficient of variation for these assays ranged from 2.1 to $4.8 \%$.

\section{Statistical analysis}

Only participants who had at least one diet recall at each study visit were included in the analyses for this report $(N=756)$. Initial analyses found that all treatment groups changed GI and GL similarly; thus groups were combined to examine overall associations of change and change in CVD risk factors. PROC MIXED model (SAS program v9.1, SAS Inc. Cary, NC) was used to examine the association of changes in GI or GL with changes in body weight and CVD risk factors including BP, lipids, fasting insulin, glucose and homocysteine. Adjusted effect estimates (beta coefficient) for each risk factor and the associated $p$ values are presented. Baseline characteristics including age, gender, race, education, cohort, site, and weight, energy intake, carbohydrate, fat, fiber intakes were included in the analyses as covariates. In addition, for risk factors for which we observed a significant association with GI or GL at either
6 or 18 months, we additionally fit a model that included interaction terms with age, gender and race. A $p$ value of $<0.05$ was considered statistically significant.

\section{Results}

Of the 756 randomized participants that were included in this report, $62 \%$ were female, $34 \%$ were African American, $64 \%$ were non-Hispanic white and $2 \%$ were other races. At baseline, these participants averaged $50.0 \pm 0.3$ (mean $\pm \mathrm{SD}$ ) years old and their average weight was $95.3 \pm 0.7 \mathrm{~kg}(210 \pm 1.5 \mathrm{lb})$. Table 1 contains the mean baseline intakes of energy, percent kcal from carbohydrate, protein, fats, blood lipid values and the changes of these variables by quartiles of GI change at 6 and 18 months. After 6 months of intervention, all groups reduced GI and GL values although the GI reduction was quite small. The reduction remained until 18 months. In addition, both GI and GL changes were significantly and positively associated with changes in dietary intakes of total fat, monounsaturated fat, saturated fat, energy and fiber but inversely with changes in protein intake at both 6 and 18 months (all $p<0.05$, supplemental table). Both GI and GL changes were significantly associated with changes in polyunsaturated fat intake at 6 months but only the association with GL remained significant at 18 months. Change in carbohydrate intake was significantly and inversely associated with GI and GL changes at both time points but with GI change at 18 months only.

Overall, GI and GL changes were not found to be associated with body weight, BP, high density lipoporteincholesterol (HDL-C), triglycerides, or insulin, at either 6 or 18 months (Table 2). At 6 months, a significant race interaction for GI change with low density lipoproteincholesterol (LDL-C) $(p=0.04, \beta=8.51 \pm 4.21 \mathrm{mg} / \mathrm{dL}$ or $0.22 \pm 0.11 \mathrm{mmol} / \mathrm{L}$ ) was observed, however, no significant association was found when further analyses were conducted within separate racial groups. At 18 months, the change in GI was significantly and positively associated with a change in total cholesterol (TC) $(p<0.05$, $\beta=23.8 \pm 12.1 \mathrm{mg} / \mathrm{dL}$ or $0.62 \pm 0.31 \mathrm{mmol} / \mathrm{L})$ and there was a significant age interaction $(p=0.01, \beta=-0.60 \pm$ $0.23 \mathrm{mg} / \mathrm{dL}$ or $-0.02 \pm 0.01 \mathrm{mmol} / \mathrm{L}$ ). The greater the reduction in GI was, the greater the decrease in TC. In addition, the older the participants were, the lesser the impact of GI changes on TC changes.

At 18 months, the change in GL was significantly and positively associated with a change in TC $(p=0.02$, $\beta=0.28 \pm 0.15 \mathrm{mg} / \mathrm{dL}$ or $0.01 \pm 0.00 \mathrm{mmol} / \mathrm{L})$ but inversely with LDL-C $((p=0.03, \beta=-0.01 \pm 0.00 \mathrm{mg} / \mathrm{dL}$ or $-0.00 \pm 0.00 \mathrm{mmol} / \mathrm{L})$, and these associations were significantly dependent on age (Table 2). The greater the decrease in GL, the greater the reduction in TC but the lesser the reduction in LDL-C. Furthermore, the older the participants were, the lesser the impact of GL on LDL-C changes. At 6 months, the GI change was weakly 
Table 1. Baseline and changes of dietary intakes and lipid profiles by quartiles of GI change at 6 and 18 months $^{\mathrm{a}}$

\begin{tabular}{|c|c|c|c|c|c|c|c|c|c|}
\hline \multirow{2}{*}{$\begin{array}{l}\text { Mean } \pm S D \\
(N)\end{array}$} & \multirow[b]{2}{*}{ Baseline } & \multicolumn{4}{|c|}{ 6-month quartile } & \multicolumn{4}{|c|}{ I8-month quartile } \\
\hline & & I (I72) & $2(172)$ & $3(173)$ & $4(172)$ & I (182) & $2(182)$ & $3(183)$ & $4(182)$ \\
\hline GI $\Delta$ & $60 \pm 1$ & $-4.08 \pm 0.43$ & $-3.43 \pm 0.11$ & $-3.07 \pm 0.10$ & $-2.64 \pm 0.20$ & $-3.65 \pm 0.47$ & $-2.96 \pm 0.12$ & $-2.59 \pm 0.10$ & $-2.14 \pm 0.22$ \\
\hline Energy $\Delta$, Mjoule & $8.16 \pm 2.61$ & $-2.83 \pm 2.78$ & $-1.50 \pm 1.89$ & $-0.45 \pm 1.86$ & $0.62 \pm 1.85$ & $-3.14 \pm 2.52$ & $-1.23 \pm 1.92$ & $-0.3 \pm 1.87$ & $0.72 \pm 1.70$ \\
\hline Carbohydrate $\Delta, \mathrm{E} \%^{\mathrm{b}}$ & $51.1 \pm 9.6$ & $4.52 \pm 10.87$ & $5.14 \pm 10.92$ & $4.04 \pm 10.88$ & $2.44 \pm|3.2|$ & $3.31 \pm 10.37$ & $3.56 \pm 10.43$ & $1.89 \pm 10.59$ & $2.27 \pm \mid 3.31$ \\
\hline Protein $\Delta, \mathrm{E} \%$ & $15.9 \pm 4.0$ & $1.89 \pm 4.52$ & $1.20 \pm 4.08$ & $1.65 \pm 5.05$ & $0.88 \pm 6.26$ & $2.0 \pm 4.46$ & $1.84 \pm 4.75$ & $1.46 \pm 5.13$ & $1.12 \pm 5.82$ \\
\hline Total Fat $\Delta, \mathrm{E} \%$ & $33.2 \pm 7.6$ & $-5.87 \pm 8.85$ & $-5.53 \pm 9.64$ & $-4.69 \pm 9.42$ & $-3.16 \pm 10.77$ & $-4.83 \pm 9.05$ & $-4.55 \pm 8.61$ & $-2.80 \pm 9.17$ & $-2.86 \pm 10.81$ \\
\hline SFA $\Delta, \mathrm{E} \%$ & $11.0 \pm 3.2$ & $-1.98 \pm 3.94$ & $-2.11 \pm 4.20$ & $-1.66 \pm 3.75$ & $-1.16 \pm 4.43$ & $-1.62 \pm 3.65$ & $-2.05 \pm 3.58$ & $-1.04 \pm 3.81$ & $-1.47 \pm 3.99$ \\
\hline PFA $\Delta$, E\% & $6.8 \pm 2.5$ & $-1.12 \pm 3.05$ & $-1.03 \pm 2.78$ & $-0.88 \pm 2.91$ & $-0.39 \pm 3.30$ & $-0.66 \pm 3.49$ & $-0.66 \pm 3.15$ & $-0.83 \pm 2.85$ & $-0.01 \pm 3.49$ \\
\hline MFA $\Delta$, E\% & $12.7 \pm 3.4$ & $-2.54 \pm 4.12$ & $-2.13 \pm 4.27$ & $-1.94 \pm 4.18$ & $-1.43 \pm 4.79$ & $-2.26 \pm 4.35$ & $-1.69 \pm 3.99$ & $-0.88 \pm 4.43$ & $-1.20 \pm 4.87$ \\
\hline Dietary cholesterol $\Delta, \mathrm{mg}$ & $276 \pm 161$ & $-118.9 \pm 252.2$ & $-61.42 \pm|8| .3$ & $-30.45 \pm 167.4$ & $3.87 \pm 149.3$ & $-119.6 \pm 224.6$ & $-50.82 \pm 178.6$ & $-9.4 I \pm 166.4$ & $-6.69 \pm 145.3$ \\
\hline Total Chol $\Delta, \mathrm{mmol} / \mathrm{L}$ & $5.48 \pm 1.0$ & $-0.31 \pm 0.66$ & $-0.29 \pm 0.68$ & $-0.30 \pm 0.58$ & $-0.22 \pm 0.66$ & $-0.44 \pm 0.92$ & $-0.32 \pm 0.83$ & $-0.30 \pm 0.75$ & $-0.40 \pm 0.87$ \\
\hline $\mathrm{LDL}$ chol $\Delta, \mathrm{mmol} / \mathrm{L}$ & $3.50 \pm 0.89$ & $-0.18 \pm 0.56$ & $-0.24 \pm 0.57$ & $-0.24 \pm 0.52$ & $-0.20 \pm 0.60$ & $-0.40 \pm 0.87$ & $-0.32 \pm 0.82$ & $-0.31 \pm 0.73$ & $-0.36 \pm 0.81$ \\
\hline $\mathrm{HDL}$ chol $\Delta, \mathrm{mmol} / \mathrm{L}$ & $1.26 \pm 0.34$ & $-0.01 \pm 0.15$ & $-0.02 \pm 0.17$ & $-0.03 \pm 0.16$ & $-0.03 \pm 0.16$ & $0.03 \pm 0.15$ & $0.01 \pm 0.19$ & $0.01 \pm 0.18$ & $0.01 \pm 0.18$ \\
\hline Triglyceride $\Delta, \mathrm{mmol} / \mathrm{L}$ & $1.62 \pm 1.13$ & $-0.26 \pm 0.77$ & $-0.09 \pm 0.96$ & $-0.05 \pm 0.79$ & $-0.03 \pm 0.75$ & $-0.25 \pm 0.76$ & $-0.17 \pm 0.76$ & $-0.12 \pm 0.68$ & $-0.10 \pm 0.53$ \\
\hline $\mathrm{GL} \Delta$ & $|4| \pm 42$ & $-85.44 \pm 39.15$ & $-28.86 \pm 10.21$ & $2.72 \pm 10.09$ & $40.91 \pm 17.94$ & $-88.02 \pm 40.52$ & $-28.88 \pm 9.85$ & $3.40 \pm 9.06$ & $41.30 \pm 19.34$ \\
\hline
\end{tabular}

${ }^{a}$ All values expressed are mean $\pm S D$ of changes by quartiles of $\mathrm{Gl}$ change values.

${ }^{\mathrm{b}} \mathrm{E} \%=$ Energy percent. 
Table 2. Association of changes in body weight and cardiovascular risk factors with changes in GI and GL at 6 or 18 months ${ }^{\mathrm{a}}$

\begin{tabular}{|c|c|c|c|c|c|c|c|c|}
\hline \multirow[b]{2}{*}{ Risk factor } & \multicolumn{2}{|c|}{ 6-month change in $\mathrm{Gl}$} & \multicolumn{2}{|c|}{ I8-month change in $\mathrm{Gl}$} & \multicolumn{2}{|c|}{ 6-month change in $\mathrm{GL}$} & \multicolumn{2}{|c|}{ I8-month change in GL } \\
\hline & $\beta \pm S E$ & $P$ value & $\beta \pm S E$ & $P$ value & $\beta \pm S E$ & $P$ value & $\beta \pm S E$ & $P$ value \\
\hline Body weight, kg & $-4.28 \pm 4.75$ & 0.37 & $5.88 \pm 5.77$ & 0.31 & $-0.05 \pm 0.06$ & 0.39 & $0.05 \pm 0.07$ & 0.53 \\
\hline SBP, mmHg & $1.08 \pm 4.31$ & 0.80 & $2.95 \pm 4.80$ & 0.54 & $0.05 \pm 0.06$ & 0.38 & $0.02 \pm 0.06$ & 0.78 \\
\hline DBP, mmHg & $1.82 \pm 3.01$ & 0.55 & $0.62 \pm 3.27$ & 0.85 & $0.04 \pm 0.04$ & 0.28 & $0.00 \pm 0.04$ & 0.99 \\
\hline $\mathrm{TC}, \mathrm{mmol} / \mathrm{L}^{\mathrm{b}}$ & $0.27 \pm 0.32$ & 0.41 & $0.62 \pm 0.31$ & $0.05^{\mathrm{c}}$ & $0.00 \pm 0.00$ & 0.92 & $0.01 \pm 0.00$ & $0.02^{\mathrm{d}}$ \\
\hline LDL-C, mmol/L & $0.08 \pm 0.30$ & $0.79^{\mathrm{e}}$ & $0.28 \pm 0.29$ & 0.10 & $0.00 \pm 0.00$ & 0.74 & $-0.00 \pm 0.00$ & $0.03^{d}$ \\
\hline HDL-C, mmol/L & $0.05 \pm 0.08$ & 0.54 & $0.15 \pm 0.09$ & 0.09 & $0.00 \pm 0.00$ & 0.42 & $0.00 \pm 0.00$ & 0.18 \\
\hline Triglyceride $\mathrm{mmol} / \mathrm{L}$ & $0.35 \pm 0.41$ & 0.40 & $0.59 \pm 0.36$ & 0.10 & $-0.01 \pm 0.01$ & 0.20 & $0.00 \pm 0.00$ & 0.96 \\
\hline Fasting insulin, pmol/L & $17.4 \pm 26.4$ & 0.51 & $45.5 \pm 35.5$ & 0.20 & $0.24 \pm 0.33$ & 0.48 & $0.27 \pm 0.44$ & 0.54 \\
\hline Fasting glucose, $\mathrm{mmol} / \mathrm{L}$ & $-0.68 \pm 0.37$ & 0.06 & $-0.25 \pm 0.40$ & 0.53 & $-0.00 \pm 0.01$ & 0.37 & $-0.00 \pm 0.01$ & 0.58 \\
\hline Homocysteine, $\mu \mathrm{mol} / \mathrm{L}$ & $-7.89 \pm 4.33$ & 0.07 & NA & NA & $-0.01 \pm 0.06$ & 0.88 & NA & NA \\
\hline
\end{tabular}

${ }^{a}$ All models were adjusted for energy intake, percent kcal from carbohydrate, percent kcal from fat, fiber, age, gender, race, education, treatment and cohort. $\beta$ : adjusted effect estimates, TC: total cholesterol, LDL-C: Low density lipoprotein cholesterol, HDL-C: high density lipoprotein cholesterol.

'Multiplication factors used to convert the conventional unit to the SI units: TC 0.0259 ; LDL-C 0.0259; HDL-C 0.0259; triglyceride 0.01 I3; insulin 6.945; glucose 0.0555 ; and homocysteine 7.397.

'The $p$ value was $<0.05$ but rounded to 0.05 for consistent presentation. The interaction term $\mathrm{Gl} \times$ age was statistically significant at $18 \mathrm{months}$ for TC $(p=0.0 \mathrm{I}, \beta=-0.02 \pm 0.0 \mathrm{I} \mathrm{mmol} / \mathrm{L})$

${ }^{\mathrm{d}}$ The interaction term GL $\times$ age was statistically significant at 18 months for TC $(p=0.03, \beta=-0.00 \pm 0.00 \mathrm{mmol} / \mathrm{L})$ and LDL-C $(p=0.03, \beta=-0.0 \pm 0.0 \mathrm{mmol} / \mathrm{L})$.

${ }^{\mathrm{e}}$ The interaction term GI $\times$ Race was statistically significant at 6 months for LDL-C ( $\left.p=0.04, \beta=0.22+0.1 \mathrm{I} \mathrm{mmol} / \mathrm{L}\right)$, however, when the same model was analyzed for the two racial groups separately, neither showed a significant association. 
associated with changes in fasting glucose and homocysteine level in an inverse direction $(p=0.06$ and 0.07 , respectively).

\section{Discussion}

Overall, the finding from this study suggests that GI and GL changes are associated with TC and LDL changes. In addition, the older the participants, the lesser the impact of GL changes on TC and LDL-C. In other words, the older the participant, a greater increase in GL was associated with a smaller increase in TC and a smaller decrease in LDL-C. This is consistent with a previous study which showed that younger participants were expected to reduce LDL-C more than older participants did in response to weight loss (21). It is, however, unclear why the association of GL with TC and LDL-C was in opposite direction.

Although several previous studies have found an association between GI and/or GL with some CVD risk factors (2-4), not all studies observed an association (5-6). Similarly, a recent meta-analysis of observational studies reported that a low GI or GL diet is associated with a reduced risk of diabetes and heart disease (10), but other reports did not find an association (12, 22, 23). In a small cross-sectional study (2) of 32 Japanese women, dietary GI and GL were positively associated with triacylglycerol and negatively with HDL-C. Likewise, using data from 13,907 participants in the NHANES III survey and 7,321 Caucasian Whitehall II participants $(4,6)$, a higher GI and GL were found to be associated with a lower HDL-C. In another study of 1,354 Japanese farmers, Murakami reported the following: a positive association between GI and BMI, triacylglycerol, and fasting glucose; a significant and positive association between GL and triacyglyerol and fasting glucose; and a negative association between GL and HDL-C (24). However, in three other studies $(23,25)$, no clear links were detected between GI or GL with body weight or other CVD risk factors.

When the impact of GI and/or GL on CVD risk factors was examined in randomized controlled trials, results have similarly been inconsistent. A low GI and/or GL diet was found to increase weight loss in the short term (26), and to decrease both LDL-C (27) and triacylglyerol (28), while other investigators reported that low GI and/or GL diets had no impact on weight loss or CVD risk factors $(5,8)(28,29)$. A review of 14 earlier intervention studies, however, reported that a low GI diet often resulted in lower triglycerides, LDL-C and TC to HDL-C ratios (30). It is possible that if GI or GL is associated with any of the CVD risk factors, it exists in certain subgroups only (i.e. men, certain age groups) (31). One study (32) found that the association between GL and triacylglycerol was nearly four times greater among women with $\mathrm{BMI} \geq 25$ than among those with $\mathrm{BMI}<25$. Another reported that GI was directly related to TC changes in men and to LDL-C in women and both relationships were modified by age, stronger for younger than for older participants (31). A recent study also showed that increasing high-GI foods and increasing GL were significantly associated with an increase in all risk for CHD in women only but not men (33). Indeed, older individuals may have a slower initial glycemic response as compared to younger individuals (34). These observations are consistent with the current findings of a significant age interaction for the association between GI and TC and between GL and TC and LDL-C.

The finding of an inverse association between GI change and homocysteine level is consistent with previous findings that plasma homocysteine was directly associated with insulin resistance and a low GI diet tended to decrease homocysteine level (35). It was hypothesized that a high GI diet may increase homocysteine by inhibiting hepatic expression of cystathionine b-synthase which catalyzes the transsulfuration from homocysteine to cystathionine (15).

A potential explanation for the association of GI to CVD risk factors may relate to its potential contribution to insulin resistance (3). Consumption of high GI or GL diets long term accumulatively may contribute to aggravated postprandial glycemia and subsequently insulin resistance and disordered lipid profile. Although this study did not find a significant association between GI or GL and fasting glucose or insulin levels, it is possible that the high glycemic effect of a high GI or GL diet may affect serum lipids through, at least in part, its impact on insulin and its lipogenic effect (30).

Since energy intake was controlled for in all analytical models and both GI and GL changes traveled closely with changes in energy intake, it is possible that any potential association between GI and/or GL and the risk factors may have been masked by the impact of energy intake on the risk factors. The possibility of underreporting in dietary intake may also have impacted the classification of GI and/or GL and thus the association with the risk factors.

This study cannot exclude the possibility that an association between GI and/or GL and CVD risk factors may truly be non-existent. The current study design, sample size and/or data collection structure may also have limited the detection of the association. Indeed, a recent report shows that the short term glycemic response ( $2 \mathrm{~h}$ as in the case of GI or GL) may not represent the long term glycemic impact (36). In addition, different types of carbohydrate (such as fructose and glucose) may have different metabolic effects. Some have suggested that a fructose index may be more relevant for CVD risk than GI (37). Since the PREMIER study was not designed to examine this question initially, there is the possibility of 
lack of power or proper measurement methodology. Thus, the results of this study should be viewed as exploratory for hypotheses-generating and interpreted with caution and be verified with future independent studies. Other limitations of the study include inherent issues with all dietary data collections and the fact that two 24-h dietary recalls may not capture usual intake of the population. However, the assessment methodologies of dietary intake and GI and GL values used in this study are commonly adopted by other studies and are similar to the studies reviewed above.

Further, it is unclear how the ranges of GI and GL examined in previous studies affected associations with CVD risk factors. When comparing to previous studies, the mean GI and GL values ( 60/140) observed in the current study can be considered either low (32) or high (3). How such discrepancy in defining a high or low GI or GL value may explain the observed inconsistent findings is not clear. Nevertheless, it is possible that if a relationship between GI or GL and CVD factors exists, it exists in a continuous fashion across a wide range of GI and GL values and this is how it was examined in this report.

Although the PREMIER intervention was not specifically designed to change dietary GI or GL, it is obvious that the intervention helped to reduce both. Reduction in GL could have been achieved by replacing intake of high-GI foods with low-GI foods and/or lowering of carbohydrate foods overall. These strategies may impact metabolism differently and so may have contributed to the variable results observed. The fact that participants may have reduced carbohydrate foods overall may also explain the finding that both GI and GL changes were significantly and positively associated with changes in energy intake.

Even though GI and GL concept is not officially promoted by the American Diabetes Association, this concept is supported and practiced by some diabetologists and is widely promoted by many entities in the public domain. Thus, it is important to clarify the clinical impact of GI and/or GL. In addition, since the diabetic population is especially at risk for cardiovascular events, it is important to understand more about the association between GI and GL and lipids and other CVD risk factors. As the result of this study indicates, a healthy eating intervention improves GI and GL and thus GI and GL can provide a mean to evaluate dietary quality in epidemiological studies. However, attention should be given to methodological issues related to usage of different dietary assessments and reliability of GI and GL estimates (38).

\section{Conclusion}

The current results suggest relatively weak associations between dietary GI and GL with changes in TC and
LDL-C that are evident after 18 months, but not after 6 months of the intervention. The measurement of GI and GL still holds its promise in predicting CVD risk and in refining CVD risk reduction program based on the current and other studies, further investigation into this relationship is important because of its potential clinical impact. Inclusion of GI and GL concept in dietary intervention or public health nutrition education program may enhance the quality and complement the entire intervention. In addition, clinical implementation of GI and GL may benefit from incorporating additional dietary considerations so that the resulting dietary changes may achieve the maximum potential for CVD health.

\section{Conflict of interest and funding}

The authors have not received any funding or benefits from industry or elsewhere to conduct this study.

\section{References}

1. Jenkins DJ, Wolever TM, Taylor RH, Barker H, Fielden H, Baldwin JM, et al. Glycemic index of foods: a physiological basis for carbohydrate exchange. Am J Clin Nutr 1981; 34: 362-6.

2. Amano Y, Kawakubo K, Lee JS, Tang AC, Sugiyama M, Mori K. Correlation between dietary glycemic index and cardiovascular disease risk factors among Japanese women. Eur J Clin Nutr 2004; 58: 1472-8.

3. Beulens JW, de Bruijne LM, Stolk RP, Peeters PH, Bots ML, Grobbee DE, et al. High dietary glycemic load and glycemic index increase risk of cardiovascular disease among middle-aged women: a population-based follow-up study. J Am Coll Cardiol 2007; 50: 14-21.

4. Mosdol A, Witte DR, Frost G, Marmot MG, Brunner EJ. Dietary glycemic index and glycemic load are associated with high-density-lipoprotein cholesterol at baseline but not with increased risk of diabetes in the Whitehall II study. Am J Clin Nutr 2007; 86: 988-94.

5. van Dam RM, Visscher AW, Feskens EJ, Verhoef $P$, Kromhout D. Dietary glycemic index in relation to metabolic risk factors and incidence of coronary heart disease: the Zutphen Elderly Study. Eur J Clin Nutr 2000; 54: 726-31.

6. Ford ES, Liu S. Glycemic index and serum high-density lipoprotein cholesterol concentration among us adults. Arch Intern Med 2001; 161: 572-6.

7. Nansel TR, Gellar L, McGill A. Effect of varying glycemic index meals on blood glucose control assessed with continuous glucose monitoring in youth with type 1 diabetes on basal-bolus insulin regimens. Diabetes care 2008; 31: 695-7.

8. Das SK, Gilhooly CH, Golden JK, Pittas AG, Fuss PJ, Cheatham RA, et al. Long-term effects of 2 energy-restricted diets differing in glycemic load on dietary adherence, body composition, and metabolism in CALERIE: a 1-y randomized controlled trial. Am J Clin Nutr 2007; 85: 1023-30.

9. Sloth B, Krog-Mikkelsen I, Flint A, Tetens I, Bjorck I, Vinoy S, et al. No difference in body weight decrease between a lowglycemic-index and a high-glycemic-index diet but reduced LDL cholesterol after 10-wk ad libitum intake of the low-glycemicindex diet. Am J Clin Nutr 2004; 80: 337-47.

10. Barclay AW, Petocz P, McMillan-Price J, Flood VM, Prvan T, Mitchell P, et al. Glycemic index, glycemic load, and chronic 
disease risk - a meta-analysis of observational studies. Am J Clin Nutr 2008; 87: 627-37.

11. Hodge AM, English DR, O'Dea K, Giles GG. Glycemic index and dietary fiber and the risk of type 2 diabetes. Diabetes care 2004; 27: 2701-6.

12. Meyer KA, Kushi LH, Jacobs DR, Jr, Slavin J, Sellers TA, Folsom AR. Carbohydrates, dietary fiber, and incident type 2 diabetes in older women. Am J Clin Nutr 2000; 71: 921-30.

13. Stevens J, Ahn K, Juhaeri, Houston D, Steffan L, Couper D. Dietary fiber intake and glycemic index and incidence of diabetes in African-American and white adults: the ARIC study. Diabetes care 2002; 25: 1715-21.

14. Beer C, Alfonso H, Flicker L, Norman PE, Hankey GJ, Almeida OP. Traditional risk factors for incident cardiovascular events have limited importance in later life compared with the health in men study cardiovascular risk score. Stroke 2011; 42: 952-9.

15. McCarty MF. Insulin secretion as a potential determinant of homocysteine levels. Medical Hypotheses 2000; 55: 454-5.

16. Funk KL, Elmer PJ, Stevens VJ, Harsha DW, Craddick SR, Lin PH, et al. PREMIER - A trial of lifestyle interventions for blood pressure control: intervention design and rationale. Health Promotion Practice 2008; 9: 271-80.

17. Appel LJ, Champagne CM, Harsha DW, Cooper LS, Obarzanek E, Elmer PJ, et al. Effects of comprehensive lifestyle modification on blood pressure control: main results of the PREMIER clinical trial. JAMA 2003; 289: 2083-93.

18. Flood A, Subar AF, Hull SG, Zimmerman TP, Jenkins DJ, Schatzkin A. Methodology for adding glycemic load values to the National Cancer Institute Diet History Questionnaire Database. J Am Diet Assoc 2006; 106: 393-402.

19. Willett WC, Howe GR, Kushi LH. Adjustment for total energy intake in epidemiologic studies. Am J Clin Nutr 1997; 65: 1220S-8S.

20. Svetkey LP, Harsha DW, Vollmer WM, Stevens VJ, Obarzanek E, Elmer PJ, et al. Premier: a clinical trial of comprehensive lifestyle modification for blood pressure control: rationale, design and baseline characteristics. Ann Epidemiol 2003; 13: $462-71$.

21. Dattilo AM, Kris-Etherton PM. Effects of weight reduction on blood lipids and lipoproteins: a meta-analysis. Am J Clin Nutr 1992; 56: 320-8.

22. Levitan EB, Mittleman MA, Hakansson N, Wolk A. Dietary glycemic index, dietary glycemic load, and cardiovascular disease in middle-aged and older Swedish men. Am J Clin Nutr 2007; 85: 1521-6.

23. Liese AD, Schulz M, Fang F, Wolever TM, D'Agostino RB, Jr, Sparks KC, et al. Dietary glycemic index and glycemic load, carbohydrate and fiber intake, and measures of insulin sensitivity, secretion, and adiposity in the Insulin Resistance Atherosclerosis Study. Diabetes care 2005; 28: 2832-8.

24. Murakami K, Sasaki S, Takahashi Y, Okubo H, Hosoi Y, Horiguchi $\mathrm{H}$, et al. Dietary glycemic index and load in relation to metabolic risk factors in Japanese female farmers with traditional dietary habits. Am J Clin Nutr 2006; 83: 1161-9.

25. Gaesser GA. Carbohydrate quantity and quality in relation to body mass index. J Am Diet Assoc 2007; 107: 1768-80.

26. Maki KC, Rains TM, Kaden VN, Raneri KR, Davidson MH. Effects of a reduced-glycemic-load diet on body weight, body composition, and cardiovascular disease risk markers in overweight and obese adults. Am J Clin Nutr 2007; 85: 724-34.

27. McMillan-Price J, Petocz P, Atkinson F, O’Neill K, Samman S, Steinbeck K, et al. Comparison of 4 diets of varying glycemic load on weight loss and cardiovascular risk reduction in overweight and obese young adults: a randomized controlled trial. Arch Intern Med 2006; 166: 1466-75.

28. Ebbeling CB, Leidig MM, Sinclair KB, Seger-Shippee LG, Feldman HA, Ludwig DS. Effects of an ad libitum low-glycemic load diet on cardiovascular disease risk factors in obese young adults. Am J Clin Nutr 2005; 81: 976-82.

29. Frost GS, Brynes AE, Bovill-Taylor C, Dornhorst A. A prospective randomised trial to determine the efficacy of a low glycaemic index diet given in addition to healthy eating and weight loss advice in patients with coronary heart disease. Eur J Clin Nutr 2004; 58: 121-7.

30. Ludwig DS. The glycemic index: physiological mechanisms relating to obesity, diabetes, and cardiovascular disease. JAMA 2002; 287: 2414-23.

31. Oxlund AL, Heitmann BL. Glycaemic index and glycaemic load in relation to blood lipids -6 years of follow-up in adult Danish men and women. Public Health Nutr 2006; 9: 737-45.

32. Willett W, Manson J, Liu S. Glycemic index, glycemic load, and risk of type 2 diabetes. Am J Clin Nutr 2002; 76: 274S-80S

33. Sieri S, Krogh V, Berrino F, Evangelista A, Agnoli C, Brighenti $\mathrm{F}$, et al. Dietary glycemic load and index and risk of coronary heart disease in a large Italian cohort. The EPICOR Study. Arch Intern Med 2010; 170(7): 640-7.

34. Basu R, Man CD, Campioni M, Basu A, Klee G, Toffolo G, et al. Effects of age and sex on postprandial glucose metabolism. Differences in glucose turnover, insulin secretion, insulin action, and hepatic insulin extraction. Diabetes 2006; 55: 2001-14.

35. Lukaczer D, DeAnn JL, Lerman RH, Darland G, Schiltz B, Tripp M, et al. Effect of a low glycemic index diet with soy protein and phytosterols on CVD risk factors in postmenopausal women. Nutrition 2006; 22: 104-13.

36. Stanhope KL, Havel PJ. Fructose consumption: considerations for future research on its effects on adipose distribution, lipid metabolism, and insulin sensitivity in humans. J Nutr 2009; 139: $1236 \mathrm{~S}-41 \mathrm{~S}$

37. Segal MS, Gollub E, Johnson RJ. Is the fructose index more relevant with regards to cardiovascular disease than the glycemic index? Eur J Nutr 2007; 46: 406-17.

38. van Bakel MME, Slimani N, Feskens EJM, Du H, Beulens JWJ, van der Schouw YT, et al. Methodological challenges in the application of the glycemic index in epidemiological studies using data from the European prospective investigation into canter and nutrition. J Nutr 2009; 139: 568-75.

\footnotetext{
*Pao-Hwa Lin

Department of Medicine

Nephrology Division

Box 3487

DUMC, Durham

NC 277I0

USA

Tel: + I 919-660-6685

Fax: +1 919-660-8802

Email: pao.hwa.lin@dm.duke.edu
} 